\title{
Development of $28 \mathrm{GHz}$ and $77 \mathrm{GHz}$, Mega-Watt Gyrotrons for Fusion Devices
}

\author{
T. Kariya ${ }^{1}$, R. Minami ${ }^{1}$, T. Imai ${ }^{1}$, M. Ota ${ }^{1}$, Y. Endo ${ }^{1}$, \\ S. Kubo ${ }^{2}$, T. Shimozuma ${ }^{2}$, H. Takahashi' ${ }^{2}$, Y. Yoshimura ${ }^{2}$, S. Ito ${ }^{2}$, T. Mutoh', \\ K. Sakamoto ${ }^{3}$, Y. Mitsunaka ${ }^{4}$ \\ ${ }^{1}$ Plasma Research Center (PRC), University of Tsukuba, Tsukuba, Ibaraki, 305-8577, \\ Japan: \\ ${ }^{2}$ National Institute for Fusion Science (NIFS), Toki, Gifu, 509-5292, Japan: \\ ${ }^{3}$ Japan Atomic Energy Agency (JAEA), Naka, Ibaraki, 311-0193, Japan: \\ ${ }^{4}$ Toshiba Electron Tube \& Devices Co.,Ltd. (TETD), Otawara, Tochigi, 324-8550, Japan: \\ Email : kariya@prc.tsukuba.ac.jp
}

\begin{abstract}
A $28 \mathrm{GHz} 1 \mathrm{MW}$ with $\mathrm{TE}_{8,3}$ cavity for GAMMA10 tandem mirror and a 77 $\mathrm{GHz}$ 1.5 MW gyrotron with $\mathrm{TE}_{18,6}$ for Large Helical Device (LHD) have been developed to upgrade their Electron Cyclotron Heating $(\mathrm{ECH})$ systems. In the $28 \mathrm{GHz}$ gyrotron, the maximum power of $1.05 \mathrm{MW}$ was obtained, which is in agreement with its design target value. And the high efficiency of $40 \%$ without collector potential depression (CPD) was obtained with $0.8 \mathrm{MW}$. In the first and second $77 \mathrm{GHz}$ gyrotrons, $0.8 \mathrm{MW} 3.6$ sec., 0.3 MW 60 sec. operations have been achieved, but several issues due to the stray RF and velocity dispersion have been found, too. In consideration of these, the design improvement was performed to aim at $1.5 \mathrm{MW}$ in the third tube. In the short pulse test, the maximum output power of $1.6 \mathrm{MW}$ and the maximum total efficiency of $49.4 \%$ with CPD were obtained. In the long pulse test, the pulse length extended to $5 \mathrm{sec}$. with $1 \mathrm{MW}$, $1800 \mathrm{sec}$. with $0.1 \mathrm{MW}, 1.6 \mathrm{sec}$. with 1.5 MW. Total injection power of 3.1 MW to LHD plasma has been achieved. It is shown that the electron beam pitch factor $\alpha$ decreases with increasing of beam current, comparing with the experimental and calculation results.
\end{abstract}

Keywords Gyrotron, MW, 28 GHz, 77 GHz, ECH, tandem mirror, GAMMA10, LHD

\section{Introduction}

The electron cyclotron heating $(\mathrm{ECH})$ is attractive and promising for heating, current drive and plasma control of toroidal magnetic confinement systems. The ECH is also essential for tandem mirror devices to achieve potential confinement and high electron temperature [1][2]. A gyrotron is a powerful and an essential tool for ECH. Recent progress of the gyrotron has widened the use of gyrotrons for fusion research [3][4]. High power and long pulse operations of the gyrotron and the efficient transmission of its output are quite important to achieve better plasma performances.

As the first step of gyrotron development for tandem mirror GAMMA10 at Plasma Research Center (PRC) in University of Tsukuba, the $28 \mathrm{GHz}$ gyrotron output power was increased from $0.2 \mathrm{MW}$ to $0.5 \mathrm{MW}$ for the higher potential and electron temperature, in the collaboration with Japan Atomic Energy Agency (JAEA) and Toshiba Electron Tube \& Devices Co.,Ltd. (TETD) [5]. Three $28 \mathrm{GHz} 0.5 \mathrm{MW}$ gyrotrons were applied to GAMMA 
10 plasma. New record values of ion confining potential and electron temperature were obtained in 2006 [6]. As the second step, the development of a $28 \mathrm{GHz}, 1 \mathrm{MW}, 1 \mathrm{sec}$. for GAMMA 10 and the $77 \mathrm{GHz} 1 \mathrm{MW}$ gyrotrons for Large Helical Device (LHD) in National Institute for Fusion Science (NIFS) is carried out under the joint program between NIFS and PRC with the collaboration of JAEA and TETD [7]. Two $77 \mathrm{GHz} 1 \mathrm{MW}$ gyrotrons \#1 and \#2 have been tested in 2007 and 2008 respectively. Typical achieved parameters of \#1 and \#2 gyrotrons until the end of 2008 are maximum MOU (matching optics unit) output power of $1.1 \mathrm{MW}$, maximum total efficiency $39.4 \%$ with collector potential depression (CPD) and long pulse operations of $0.8 \mathrm{MW} 3.6 \mathrm{sec}$., $0.3 \mathrm{MW} 60 \mathrm{sec}$. and so on. In consideration of \#1 and \#2 gyrotrons test results, the design improvement was performed for the 1.5 MW tube. The $77 \mathrm{GHz} 1.5 \mathrm{MW}$ gyrotron \#3 has been fabricated and tested in 2009. This report describes the design and the test result of $28 \mathrm{GHz} 1 \mathrm{MW}$ and $77 \mathrm{GHz} 1.5$ MW gyrotrons.

\section{Design of $28 \mathrm{GHz}$ and $77 \mathrm{GHz}$ Gyrotrons}

A set of design parameters of the $28 \mathrm{GHz} 1 \mathrm{MW}$ and the $77 \mathrm{GHz} 1.5 \mathrm{MW}$ gyrotron is shown in Table1. Both gyrotrons are designed to use the same superconducting magnet (SCM) and, therefore, the magnetic distribution.

\subsection{GHz Design}

The picture and the structural cross-section of $28 \mathrm{GHz} 1 \mathrm{MW}$ gyrotron are shown in Fig.1. The first operation target is $1 \mathrm{MW}$ output for $1 \mathrm{sec}$. limited by the window heat load. The magnetron injection gun (MIG) is a triode gun to be able to control the electron beam parameters by anode voltage, and has same cathode structure with the $77 \mathrm{GHz} 1.5 \mathrm{MW}$ gyrotron to get compatibility. An anode voltage dependences of the pitch factor $\alpha$ (= perpendicular velocity to the magnetic field / parallel velocity) and $\alpha$ spread calculated by MIG simulation code are shown in

Fig.2. It has been obtained that the pitch factor $\alpha$ are $1.1 \sim 1.2$ with $\alpha$ spread of $6 \sim 7 \%$ at the beam voltage of $80 \mathrm{kV}$ and the anode voltage $37 \sim 39 \mathrm{kV}$. An RF absorber ( $\mathrm{SiC}$ ceramics) is inserted in the beam tunnel to suppress the parasitic oscillation. The cavity mode is $\mathrm{TE}_{8,3}$. This mode selection was made by the MIG design to use the same cathode as the 77 $\mathrm{GHz} 1.5 \mathrm{MW}$ gyrotorn, to reduce current density at the cathode and to have enough magnetic strength at MIG region. In the cavity calculation results, an oscillation power of $1.44 \mathrm{MW}$ with the oscillation efficiency of $45.1 \%$ was obtained at beam voltage $\mathrm{V}_{\mathrm{k}}=80$ $k \mathrm{~V}$, beam current $\mathrm{I}_{\mathrm{c}}=40 \mathrm{~A}$ and

Table1 A set of design parameters of the $28 \mathrm{GHz}$ $1 \mathrm{MW}$ and the $77 \mathrm{GHz} 1.5 \mathrm{MW}$ gyrotrons.

\begin{tabular}{lll}
\hline & $\begin{array}{l}28 \mathrm{GHz} \text { Gyrotron } \\
\text { for PRC(Tsukuba) }\end{array}$ & $\begin{array}{l}\text { 77GHz Gyrotom } \\
\text { for LHD(NIFS) }\end{array}$ \\
\hline Frequency & $28 \mathrm{GHz}$ & $77 \mathrm{GHz}$ \\
Output Power & $1 \mathrm{MW}$ & $1.5 \mathrm{MW} \quad 0.3 \mathrm{MW}$ \\
Pulse Width & $1 \mathrm{~s}$ & $2 \mathrm{~s} \quad \mathrm{CW}$ \\
Output Efficiency & $35 \%$ (W/O CPD) & $50 \%$ (with CPD) \\
Beam Voltage & $80 \mathrm{kV}$ & $80 \mathrm{kV}$ \\
Beam Current & $40 \mathrm{~A}$ & $50 \mathrm{~A}$ \\
Heat er Voltage & $30 \mathrm{~V}$ MAX & $30 \mathrm{VAX}$ MAX \\
Heater Current & $8 \mathrm{~A}$ MAX & $8 \mathrm{~A}$ MAX \\
MIG & triode & triod \\
Cavity mode & TE8,3 & TE 18,6 \\
Mode Converter & Built-in & Built-in \\
Output mode & Gaussian like & Gaussian like \\
Output Window & Sapphire & CVD Diamond \\
& Aperture $\phi 112 \mathrm{~mm}$ & Aperture $\phi 85 \mathrm{~mm}$ \\
Collector & W/O CPD & Depressed Collector \\
\multicolumn{4}{c}{ I.D.320mm } & I.D.320mm \\
Height & Sweeping coils & Sweeping coils \\
Weight & $2413 \mathrm{~mm}$ & $3104 \mathrm{~mm}$ \\
(W/O collector coils) & 700kg & $\sim 800 \mathrm{~kg}$ \\
\hline
\end{tabular}


$\alpha=1.2$. The $\mathrm{TE}_{8,3}$ mode $\mathrm{RF}$ wave is converted to a Gaussian-like beam by a built-in quasi-optical mode converter, and the RF beam is transmitted by four pieces of mirror system to the outside of the tube through a single disk sapphire window. The total transmission efficiency from the mode converter to the output window is $94.7 \%$ and the FWHM of RF beam power is $42 \mathrm{~mm}$ at the window. The output RF beam is adjusted its profile and phase by Matching Optics Unit (MOU), and couples to a corrugated waveguide as $\mathrm{HE}_{11}$ mode. The total transmission efficiency from the mode converter to the corrugated waveguide is $90.2 \%$. An RF transmission design at $28 \mathrm{GHz}$ has difficulty in comparison with the design at $77 \mathrm{GHz}$, because of a diffraction effect caused by a long wavelength. The temperature rise of sapphire output window is calculated to be $60 \mathrm{~K}$ with $1 \mathrm{sec}$. of $1 \mathrm{MW}$ RF power. Because of thermal runaway phenomenon caused by the increase of the dielectric-loss according to the temperature rise, the pulse duration is limited to $1 \mathrm{~s}$ at the $1 \mathrm{MW}$ output. In a built-in mode converter type gyrotron, the design of the large size collector which is necessary for mega-watt gyrotron is possible because the collector does not have a role of waveguide by separating the spent electron beam and the RF beam. The collector with inner diameter of $320 \mathrm{~mm}$ has the sweep coil to reduce heat load of the spent electron beam. The sweeping frequency is $1 \sim 2 \mathrm{~Hz}$ to reduce the attenuation of the sweep magnetic field by eddy current in the collector metal surface. The collector average deposition power density is less than $0.65 \mathrm{~kW} / \mathrm{cm}^{2}$ with spent beam power of $2.5 \mathrm{MW}$.
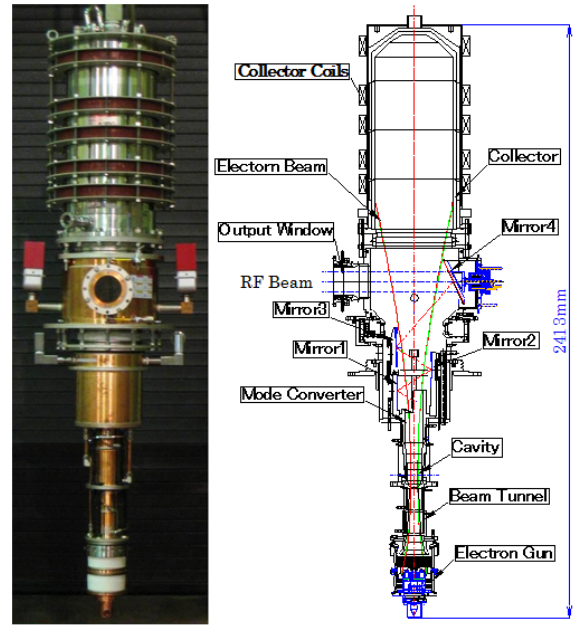

Fig.1 Picture and structural cross-section of $28 \mathrm{GHz} 1 \mathrm{MW}$ gyrotron.

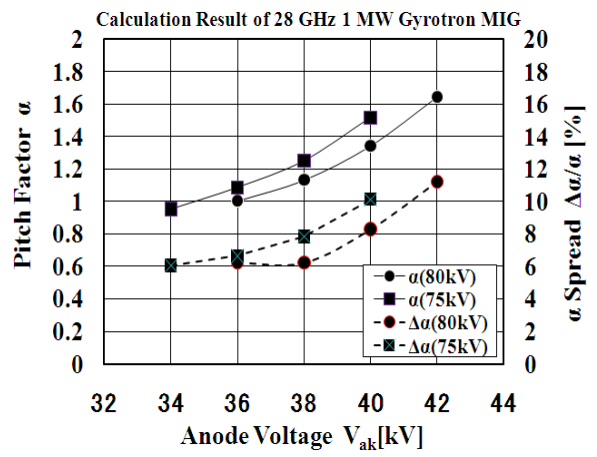

Fig.2 Anode voltage dependences of the calculated pitch factor $\alpha$ and $\alpha$ spread of $28 \mathrm{GHz} 1 \mathrm{MW}$ gyrotron.

\subsection{GHz Design}

The $77 \mathrm{GHz} 1 \mathrm{MW}$ gyrotron development for LHD in NIFS is carried out under the joint program between NIFS and PRC with the collaboration of JAEA and TETD. The first tube of $77 \mathrm{GHz}$ gyrotron was designed, taking the design essence of the ITER gyrotron which has been developing in JAEA [8][9]. Two $77 \mathrm{GHz} 1 \mathrm{MW}$ gyrotrons, \#1 and \#2 have been tested in 2007 and 2008 respectively. Major achieved parameters of \#1 and \#2 gyrotrons until the end of 2008 are maximum MOU output power of $1.1 \mathrm{MW}$, maximum 
total efficiency with collector potential depression (CPD) of $39.4 \%$, and long pulse operation of $0.8 \mathrm{MW}$ 3.6 sec., 0.3 MW 60 sec.. In consideration of \#1 and \#2 gyrotrons test results, the improvement of 1.5 MW tube design was performed for higher efficiency and higher power ECRH system. Three points of the design improvement for 1.5 MW tube are the MIG design for quality improvement of the electron beam, the cavity design for the higher oscillation efficiency and the mode converter and mirror design for improvement of the RF distribution at the output window and the reduction of the diffraction loss. The structural cross-section and the picture of $77 \mathrm{GHz}$ gyrotorn for LHD

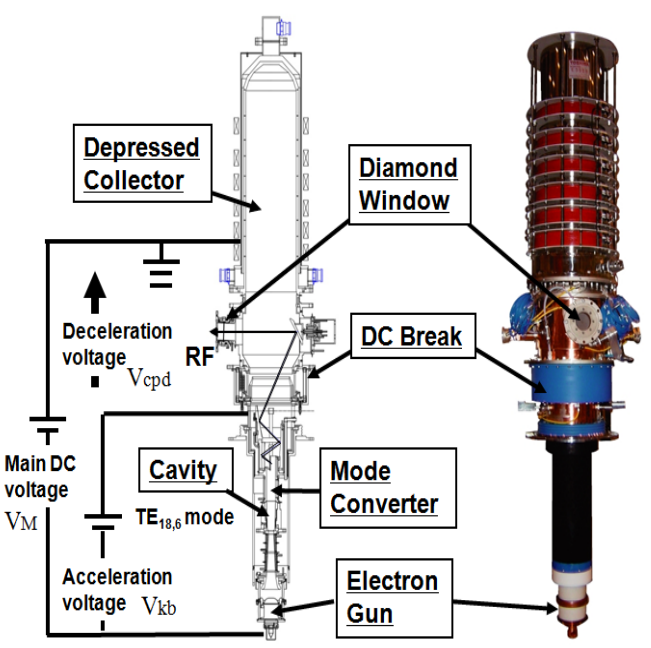

Fig.3 Structural cross-section and the picture of $77 \mathrm{GHz}$ gyrotron for LHD. (NIFS) are shown in Fig.3. The first

operation targets are 1.5 MW output in $2 \mathrm{sec}$. and $0.3 \mathrm{MW}$ in continuous wave (CW) operation. If the stray RF power which is caused by the transmission loss of RF power is small, the $\mathrm{CW}$ operation is possible at $1 \mathrm{MW}$ in the thermal designs of the cavity, the output window and the collector. The improvement points of \#3 gyrotron MIG are that the emission belt area has been enlarged for the current density decrease, and the cathode angle has been made deeper for laminar flow of electron beam in front of the cathode. The higher $\alpha$ operation with the lower $\alpha$ dispersion leading to the higher oscillation efficiency at the higher beam current will be expected by these improvements. An electron beam trajectories calculated by MIG code are shown in Fig.4. The upper figure is 1MW tube (\#1) and the lower figure is $1.5 \mathrm{MW}$ tube (\#3), respectively. The beam current density of \#3 cathode was reduced to $75.9 \%$ in comparison with that of \#1 cathode by enlarging the emission belt area. As shown in Fig4, the laminar flow of the electron beam in front of the cathode was improved by the cathode angle which has been made deeper. Anode voltage dependences of the pitch factor $\alpha$ and the $\alpha$ spread calculated by MIG simulation code are shown in Fig.5. As seen from Fig.5, a good electron beam parameter is obtained by MIG simulation, that is the pitch factor $\alpha$ are $1.1 \sim 1.2$ with small $\alpha$ spread $(<5 \%)$. An RF absorber cooled on the outer surface is inserted in the beam tunnel to suppress the parasitic oscillation. The operation mode of cavity resonator is $\mathrm{TE}_{18,6}$ which is the same as that of \#1 tube. The cavity structure was reviewed for the higher oscillation efficiency with pitch factor $\alpha$ is 1.1. The calculated $\alpha$ dependence of the cavity oscillation power and efficiency of \#3 are shown in Fig.6 in comparison with \#1 design, where the beam voltage $V_{k}$ is $80 \mathrm{kV}$ and the beam current $I_{k}$ is $50 \mathrm{~A}$. As seen from the figure, the oscillation power and efficiency of \#3 designs are higher than those of \#1 design. A calculated oscillation power of $1.42 \mathrm{MW}$ with the oscillation efficiency of $35.5 \%$ is obtained at $\alpha=1.1$. The $\mathrm{TE}_{18,6}$ mode RF wave is converted to a Gaussian-like beam by a built-in quasi-optical mode converter, and RF beam is transmitted by four pieces of mirror system to the outside of the tube through a CVD diamond window. The output RF beam is adjusted its profile and phase by MOU, and couples to a corrugated waveguide as $\mathrm{HE}_{11}$ mode. 


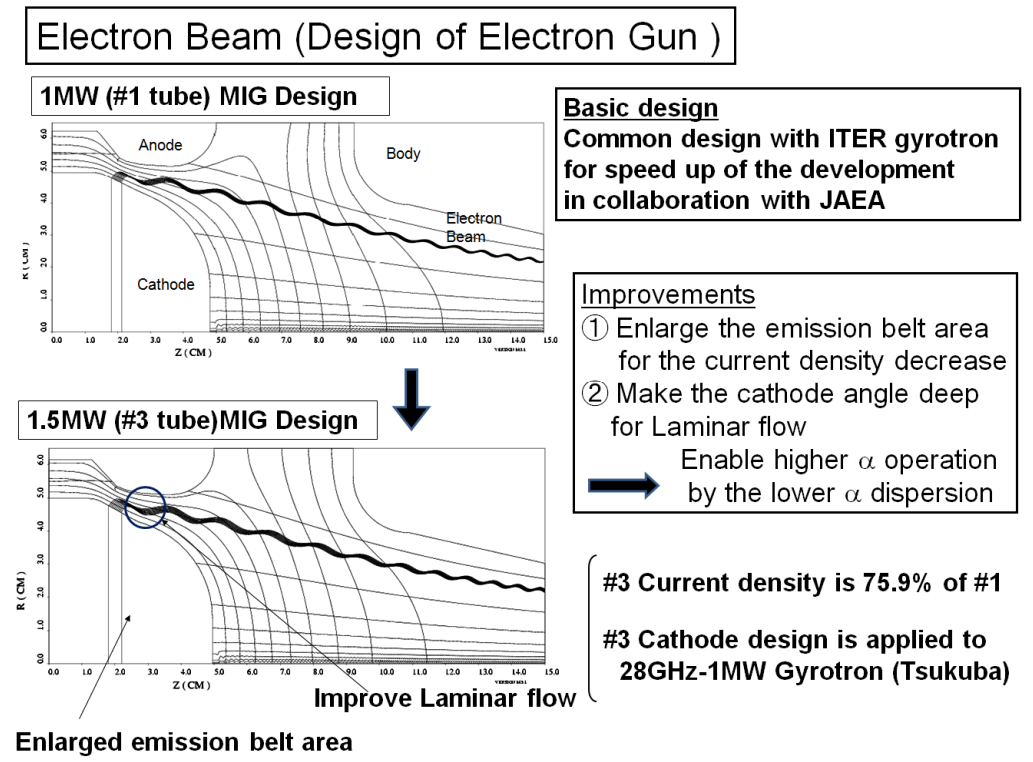

Fig.4 Electron beam trajectories of \#1 (upper) and \#3 (lower) calculated by MIG simulation code.

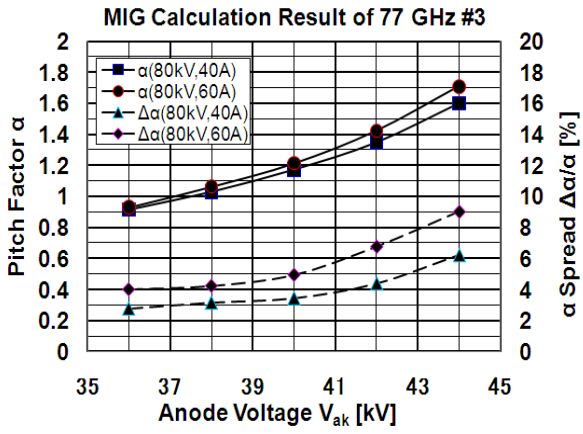

Fig.5 Anode voltage dependences of the calculated pitch factor $\alpha$ and $\alpha$ spread of $77 \mathrm{GHz} 1.5 \mathrm{MW}$ gyrotron.

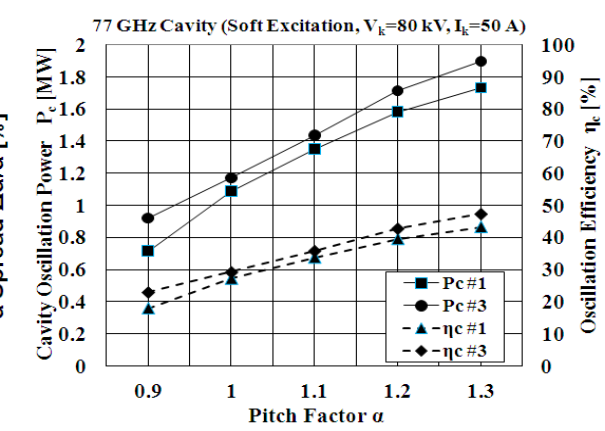

Fig.6 Alpha dependences of the cavity oscillation power and efficiency as comparison with \#3 and \#1 cavity

In the experimental test of \#1 tube, a crack on the edge of diamond disc occurred in the shot of $0.8 \mathrm{MW}$ operations. In this failure shot, the arc detector which views the output window detected the occurrence of the arc, and the high voltage power supply was cut off at 3.5 seconds. In addition, the vacuum pressure in the gyrotron deteriorated to several $10^{-5} \mathrm{~Pa}$. The response time of arc sensor is less than 3 microseconds, and the high voltage power supply is cut off within about 20 microseconds. The response time of vacuum interlock is about $0.1 \sim 0.3$ seconds. In the similar shot just before the cracked shot, 0.8 MW 3.6 sec. operations have been achieved with a little outgassing. The output RF monitor and the temperature rise of the cooling-water of each gyrotron part of the failure 
shot did not change in comparison with those of the normal shots. That is, the oscillation of the other cavity mode and the parasitic oscillation were not observed. These results led us to the conclusion that the crack was occurred by the electric discharge due to the main RF beam edge field and the stray RF field. To prevent a recurrence of the accident, several improvements were made. By making the $\mathrm{Cu}$ cover sleeve thicker, the brazing alloy used in the brazing of diamond disk was completely hidden from the RF beam line. To reduce the stray $\mathrm{RF}$ at the diamond disc edge, the $\mathrm{Cu}$ cover sleeve was coated with an RF absorber of $\mathrm{TiO}_{2}$ as shown in Fig.7. In addition, the RF distribution at the output window was improved to more axis-symmetrical distribution and the electric field at the window edge was reduced even with power up from $1 \mathrm{MW}$ to $1.5 \mathrm{MW}$ by the design change of the mode converter and the mirrors. The calculated RF distributions of \#3 and \#1 are shown in Fig.7. In comparison with \#1 design, the design of \#3 has that the RF profile is more axis-symmetric and the electric field strength at the disc edge is reduced to $\sim 1 / 3$. The calculated electric field and power density at the \#3 window edge are $458 \mathrm{~V} / \mathrm{cm}$ and 557 $\mathrm{W} / \mathrm{cm}^{2}$ at the $1 \mathrm{MW}$ output power respectively. And the total transmission efficiency from the mode converter to the corrugate waveguide has been improved slightly to $96.3 \%$ from $95.5 \%$ of \#1 design. In the gyrotron operation, some observation systems were improved. The sensitivity of the arc detector was adjusted. The temperature raise of diamond window was monitored by the IR camera installed at the MOU viewing-port. The inner surface of diamond disk was observed by a CCD camera installed at the viewing-port of gyrotron.
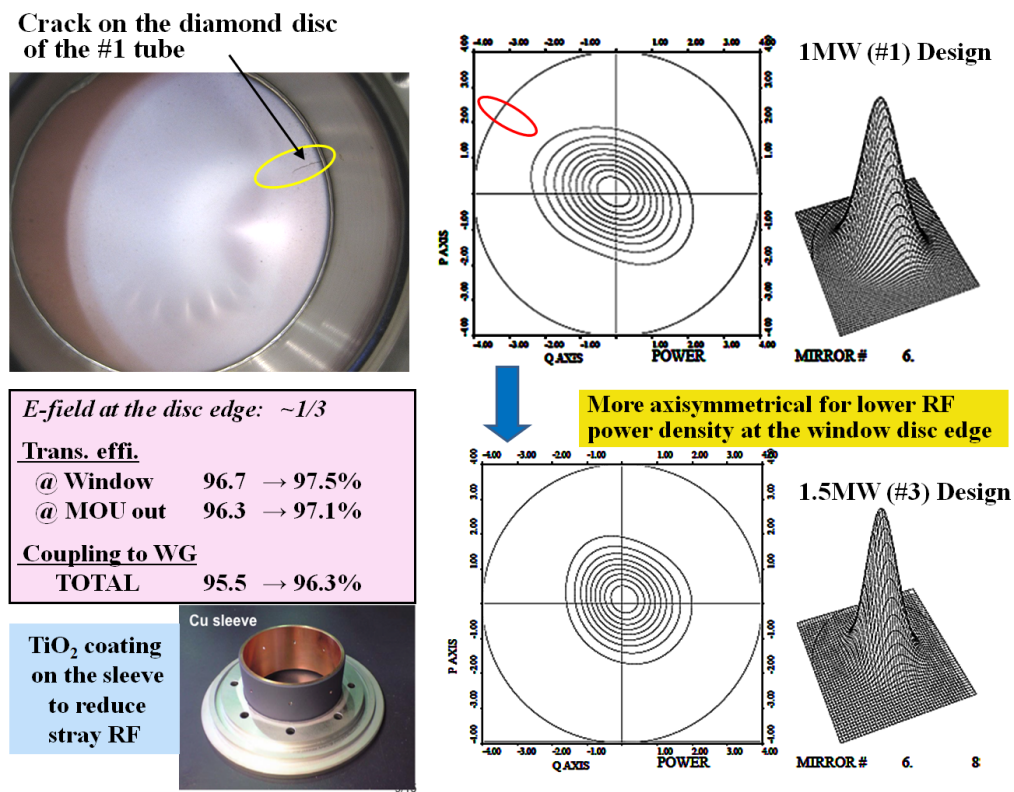

Fig.7 Design improvement of RF distribution at the output window.

The collector adopts collector potential depression (CPD) for efficiency enhancement and has the sweep coil to reduce heat load to the collector. The efficiency is enhanced by the factor of $V_{k} / V_{M}$, i.e. the total efficiency with CPD is $\eta_{\text {cpd }}=\eta_{\mathrm{o}} V_{k} / V_{M}$. Here, the $\mathrm{V}_{\mathrm{M}}\left(=\mathrm{V}_{\mathrm{k}}-\mathrm{V}_{\mathrm{cpd}}\right)$ and $\mathrm{V}$ cpd are the main power supply and CPD voltages, respectively. The $\mathrm{CPD}$ voltage is the beam deceleration voltage supplied between the body section 
(including beam tunnel, cavity, mode convertor and mirrors) and the collector and the $\eta_{\mathrm{o}}$ is the output efficiency. The collector average power deposition is under $0.55 \mathrm{~kW} / \mathrm{cm}^{2}$. The gyrotron has a DC-break section to sustain a high voltage between the body section and the collector (grounded). Likewise, the gyrotron has a body insulation jacket to sustain a high voltage between the body section and the SCM (grounded).

\section{Test Results}

\subsection{GHz Gyrotron Performance}

A $28 \mathrm{GHz} 1 \mathrm{MW}$ gyrotron has been fabricated and tested. The initial experiment with the short pulse was performed in the RF test stand of PRC in University of Tsukuba by using the SCM for $77 \mathrm{GHz} \# 3$ gyrotron.

The experimentally measured burn pattern of RF beam at output window was the Gaussian-like profile agreed with the design result. The output RF frequency was 28.04 $\mathrm{GHz}$ which is expected from the cavity design. From these experimental results, the $\mathrm{TE}_{8,3}$ mode oscillation in the cavity was confirmed. Beam current $I_{k}$ dependences of the output power $P_{o}$ and the output efficiency $\eta_{o}$ are shown in Fig.8. The $P_{o}$ is measured calorimetrically by the $\mathrm{SiC}$ water load at output window. The $\mathrm{P}_{\mathrm{o}}$ increases with increasing $\mathrm{I}_{\mathrm{k}}$ without saturation, though there are some deviations, and the output power is more than $1 \mathrm{MW}$ with 40 A. The maximum output efficiency is $40 \%$ at $\mathrm{I}_{\mathrm{k}}$ of $16 \mathrm{~A}$. The magnetic field must be optimized corresponding to each beam current value. A cavity magnetic field strength $B_{c}$ dependence of $P_{o}$ is shown in Fig.9, with $V_{k}=80 \mathrm{kV}$ and $I_{c}=40.4$ A. The $\mathrm{B}_{\mathrm{c}}$ were calculated by operation current of SCM. With $\mathrm{B}_{\mathrm{c}}$ larger than the critical magnetic field of $1.078 \mathrm{~T}$, the designed cavity oscillation mode $\mathrm{TE}_{8,3}$ was obtained. The maximum $\mathrm{P}_{\mathrm{o}}$ is obtained at the critical $\mathrm{B}_{\mathrm{c}}$. As increase in $\mathrm{B}_{\mathrm{c}}$, Po decreases. A serious mode competition was not observed.

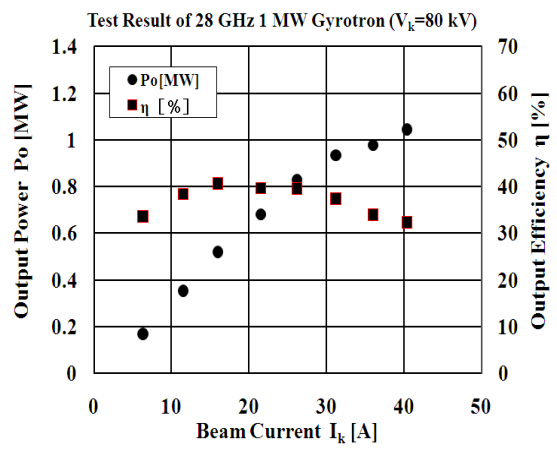

Fig.8 Beam current dependences of output power and output efficiency of 28 GHz 1 MW gyrotron.

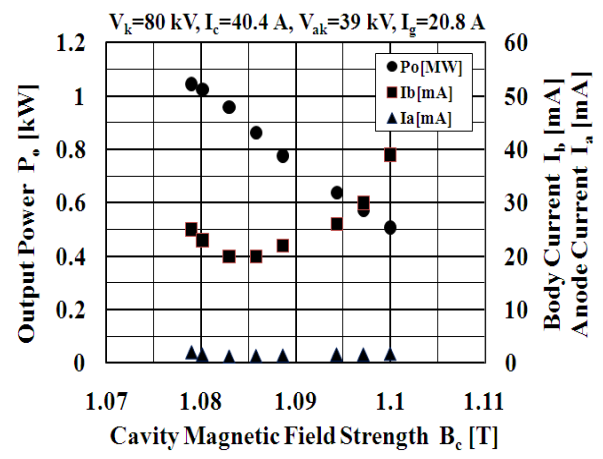

Fig.9 Cavity magnetic field strength dependences of the output power, the anode current and the body current of 28 $\mathrm{GHz} 1 \mathrm{MW}$ gyrotron.

\subsection{GHz Gyrotron Performance}

An initial $77 \mathrm{GHz}$ gyrotron test with the short pulse was performed in the RF test stand of PRC in University of Tsukuba. After confirming that short pulse performance 
didn't have a problem, gyrotron was installed to the ECH system of the LHD in NIFS, and a short pulse output adjustment and a long pulse test were carried out. The calculated RF profiles and the experimentally measured burn patterns of RF beam at output window are shown in Fig.10. The upper figure is that of $1 \mathrm{MW} \# 1$ gyrotron and the lower is that of 1.5 MW \#3. The burn pattern of RF beam well agrees with the design distribution result. In comparison with the \#1 test result, that of the \#3 has the more axis-symmetrical RF profile. The frequency of the output RF was $76.96 \mathrm{GHz}$ suggesting the oscillation of $\mathrm{TE}_{18,6}$. The burn pattern is measured by a thermo-sensible paper installed between the output window and the short pulse dummy-load. The detail RF power distribution cannot be measured by the burn pattern measurement, because a thermo-sensible paper changes into black only when exceeding a critical temperature. To measure the detail output RF beam distribution, it is the best to measure the temperature raise of the diamond window or that of the RF beam target by an IR camera. However, in the present high power test geometry, it is difficult to get a good sight of view of IR camera. As the next best, the burn pattern measurement is used and is very useful because it can be easily measured the outline of RF beam shape and its position. The position (angle) of output RF beam from the gyrotron is adjusted by moving the last inner mirror according to the result of the burn pattern measurement.
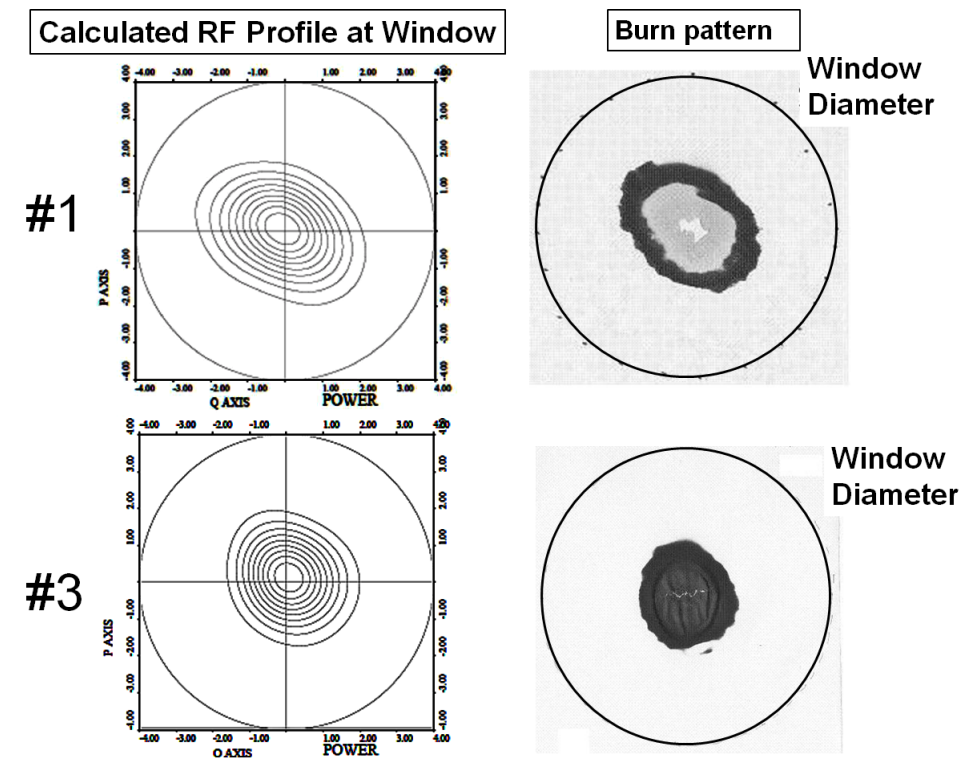

Fig.10 Calculated RF profiles and experimentally measured burn patterns of RF beam at the output window. The upper figure is the result of $\# 1$ and the lower figure is that of \#3 gyrotron.

Beam current $I_{k}$ dependences of the output power $P_{o}$ and the output efficiency $\eta_{o}$ at MOU output are shown in Fig. 11 for \#1, \#2 and \#3 gyrotrons. Each $\eta_{\mathrm{o}}$ doesn't include the efficiency enhancement by CPD. The $\eta_{\mathrm{o}}$ of \#3 was higher than those of \#1 or \#2. The $\mathrm{P}_{\mathrm{o}}$ of \#3 gyrotron increased with increasing $I_{k}$ without saturation, and more than $1.5 \mathrm{MW}$ of the output power was obtained. In the dependence of $\mathrm{P}_{\mathrm{o}}$ on cavity magnetic field strength $\mathrm{B}_{\mathrm{c}}$, a serious mode competition wasn't observed. The anode voltage $\mathrm{V}_{\mathrm{ak}}$ dependences of the $\mathrm{P}_{\mathrm{o}}$ and the anode current $\mathrm{I}_{\mathrm{a}}$ with the fixed magnetic field and the fixed beam current of 
42.1 A are shown in Fig.12. According to increase of $\alpha$ by the increase of $V_{a k}$, the oscillation of $\mathrm{TE}_{18,6}$ mode started and the $P_{o}$ saturated at $V_{a k}>39 \mathrm{kV}$. The $I_{a}$ increased rapidly with increase of $V_{a k}$, because the electrons which have the $\alpha$ more than critical value are reflected. The CPD voltage $V_{c p d}$ dependences of the $P_{o}$, the $I_{a}$, the output efficiency $\eta_{o}$ and the total efficiency $\eta_{\mathrm{t}}$ which include the efficiency enhancement by CPD are shown in Fig.13. The $P_{o}$ and the $\eta_{o}$ were almost constant in spite of the $\mathrm{V}_{\mathrm{cpd}}$ change. The $\eta_{t}$ increased as increasing of the $V_{c p d}$, and the maximum total efficiency of $50 \%$ was achieved. The $I_{a}$ which was caused by the reflected electrons by the $\mathrm{V}_{\mathrm{cpd}}$ increased slowly as increase in the $V_{\text {cpd }}$. These CPD characteristics of $P_{o}, I_{a}, \eta_{t}$, and maximum CPD voltage of the \#3 gyrotron were improved, in comparison with those of $\# 1$ and \#2. These indicate that the $\alpha$ spread of electron beam is substantially improved in \#3 gyrotron.

After the short pulse test, the long pulse test was performed. In the long pulse test, the low beam current collector conditioning was carried out first without oscillation. After the step by step conditioning, the pulse width of $1.5 \mathrm{sec}$. was achieved at the power of $1.5 \mathrm{MW}$. The time evolution of the collector sweeping coil current, beam, collector and anode voltages, beam, body and anode currents and RF output power are shown in Fig. 14. The output power decreased gradually corresponding to the decrease in the beam current. The decrease in the beam current due to the cathode cooling effect by the electron emission causes the decrease in the average output power or the termination of RF oscillation. This is one of the issues for high power long pulse (or $\mathrm{CW}$ ) operation. To avoid the decrease of the beam current, it is necessary to keep the cathode temperature constant as much as possible by the control of the cathode heater. The main achieved parameters of the \#1, \#2 and \#3 gyrotrons are shown in

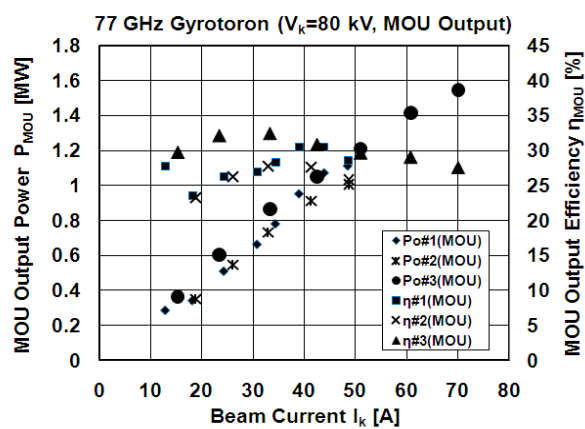

Fig.11 Beam current dependences of the MOU output power and the output efficiency of $77 \mathrm{GHz} \# 1$, \#2 and \#3 gyrotrons.

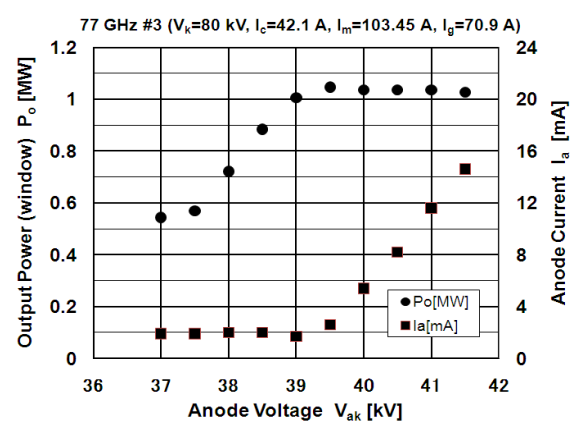

Fig.12 Anode voltage dependences of output power and anode current of 77 $\mathrm{GHz}$ 1.5 MW gyrotoron (without CPD).

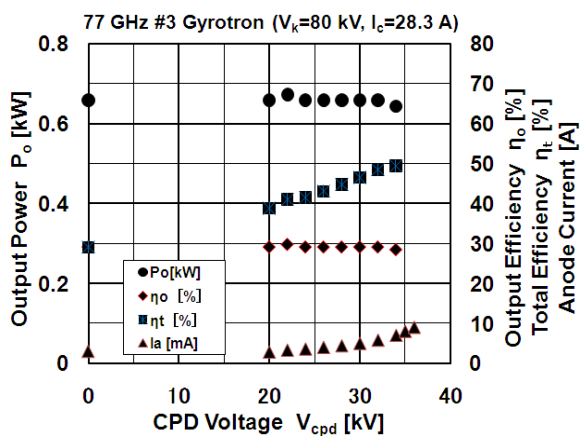

Fig. 13 CPD voltage dependences of output power, anode current, output efficiency and total efficiency which include the efficiency enhancement by CPD. 
Table2. In the long pulse operation, the output power and efficiency of \#3 were improved largely in comparison with \#1 and \#2. Both the maximum MOU output power of 1.6 MW with pulse width of $0.5 \mathrm{sec}$. and the maximum total efficiency of $49.4 \%$ with that of $2 \mathrm{~ms}$ were obtained in the \#3 gyrotron results. The other achieved long pulse parameters were 1 MW 5sec., 0.12 MW 1800 sec. and so on.

On the \#2 gyrotron operation of $0.2 \mathrm{MW}$, limitation of the pulse duration was observed around the pulse width of $300 \mathrm{sec}$. because of sudden outgassing. The cause of this outgassing is regarded as the heating of the DC break section by the stray RF which is caused by a transmission loss. On the operation of the \#3 tube which has the improved designs of mode converter and mirrors, the sudden outgassing has not been observed in $0.2 \mathrm{MW} 1200 \mathrm{sec}$. As the anode voltage $\mathrm{V}_{\mathrm{ak}}$ can be controlled at the start of $\mathrm{V}_{\mathrm{ak}}$ after improvement of the \#2 anode power supply, it is possible to apply higher $\mathrm{V}_{\mathrm{ak}}$ and CPD voltage $\mathrm{V}_{\text {cpd }}$, which has brought the output power and the total efficiency improvement. Before anode voltage control, the output power was 1.1 MW and the total efficiency was $29.8 \%$ with $\mathrm{V}_{\mathrm{ak}}=39.1 \mathrm{kV}, \mathrm{V}_{\mathrm{cpd}}=15 \mathrm{kV}$ and cavity coil current $\mathrm{I}_{\mathrm{m}}=102.4 \mathrm{~A}$. After anode voltage control, the output power was $1.3 \mathrm{MW}$ and the total efficiency was $38.2 \%$ with $\mathrm{V}_{\mathrm{ak}}=39.6 \mathrm{kV}, \mathrm{V}_{\mathrm{cpd}}=24 \mathrm{kV}$ and cavity coil current $\mathrm{I}_{\mathrm{m}}=102.08 \mathrm{~A}$. Three gyrotrons are under the long pulse test process to obtain the higher power with the longer pulse duration.

High power RF injection into the LHD plasma has been performed with the three 77 $\mathrm{GHz}$ gyrotrons [10]. The maximum total power of $3.1 \mathrm{MW}$ was injected. Some outstanding results have been obtained, such as effective local heating, density control that enables long pulse plasma sustainment. Stored energy sustained only with ECH of over $200 \mathrm{~kJ}$ that exceeds the past maximum value of $150 \mathrm{~kJ}$ in $\mathrm{ECH}$ only discharge was obtained with electron density $2.5 \times 10^{19} \mathrm{~m}^{-3}$ and ECH power $2 \mathrm{MW}$. Electron temperature higher than $10 \mathrm{keV}$ was obtained in low density experiment of $\sim 0.4 \times 10^{19} \mathrm{~m}^{-3}$. In the experiment of the $2 \mathrm{MW}$ ECH injection to NBI plasma $\left(\sim 4 \times 10^{19} \mathrm{~m}^{-3}\right)$, a suppression of electron density increase and the increase of bulk electron temperature from $1.7 \mathrm{keV}$ to $2.2 \mathrm{keV}$ were obtained.

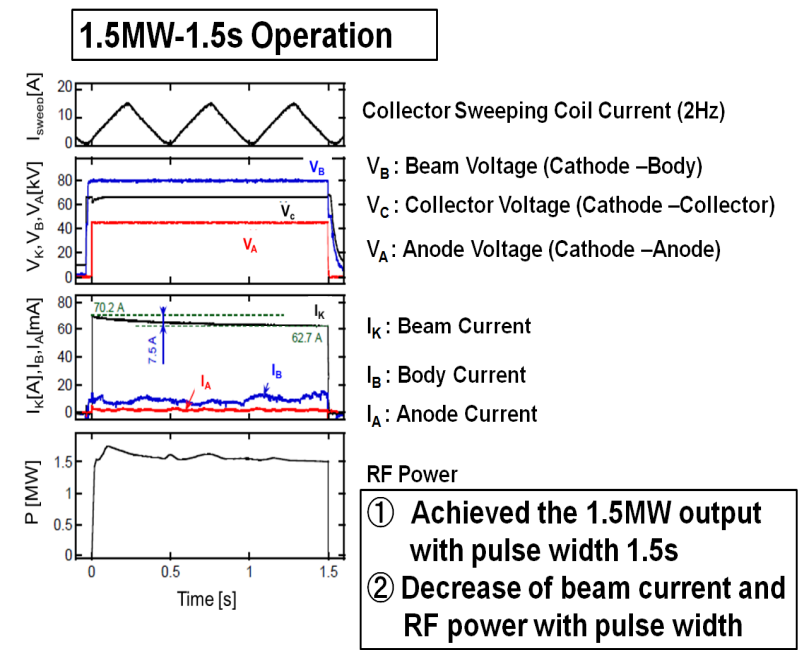

Fig.14 Time evolutions of collector sweeping coil current, beam/collector/anode voltage, beam/body/anode current, and RF output power. 
Table2 A set of main achieved parameters of three $77 \mathrm{GHz}$ gyrotrons at the end of 2009.

\begin{tabular}{|c|c|c|c|}
\hline Tube No. & Design & Short Pulse Operation & Long Pulse Operation \\
\hline$\# 1$ & $\begin{array}{c}1 \mathrm{MW} / 5 \mathrm{~s} \\
0.3 \mathrm{MW} / \mathrm{CW}\end{array}$ & $\begin{array}{l}1.11 \mathrm{MW} / 33.9 \%(28.6 \%) / 4 \mathrm{~ms} \\
1.07 \mathrm{MW} / 39.4 \%(30.5 \%) / 4 \mathrm{~ms}\end{array}$ & $\begin{array}{c}1.03 \mathrm{MW} / 32.8 \%(24.6 \%) / 5 \mathrm{~s} \\
0.31 \mathrm{MW} / 33.1 \%(24.8 \%) / 150 \mathrm{~s} \\
0.13 \mathrm{MW} / 20.6 \%(15.4 \%) / 935 \mathrm{~s}\end{array}$ \\
\hline \multirow[t]{2}{*}{ \#2 } & \multirow[t]{2}{*}{$\begin{array}{l}1.2 \mathrm{MW} / 5 \mathrm{~s} \\
0.3 \mathrm{MW} / \mathrm{CW}\end{array}$} & $\begin{array}{c}1.06 \mathrm{MW} /(27.5 \%) / 1 \mathrm{~ms} \\
0.75 \mathrm{MW} / 36.6 \%(28.4 \%) / 4 \mathrm{~ms}\end{array}$ & $\begin{array}{c}1.10 \mathrm{MW} / 29.6 \%(24.1 \%) / 1.2 \mathrm{~s} \\
1.02 \mathrm{MW} / 30.2 \%(24.6 \%) / 5 \mathrm{~s} \\
0.2 \mathrm{MW} / 19.3 \%(15.1 \%) / 370 \mathrm{~s} \\
0.11 \mathrm{MW} / 9.6 \%(8.2 \%) / 1800 \mathrm{~s}\end{array}$ \\
\hline & & Anode Voltage Control & $1.31 \mathrm{MW} / 38.2 \%(26.7 \%) / 1 \mathrm{~s}$ \\
\hline$\# 3$ & $\begin{array}{l}1.5 \mathrm{MW} / 2 \mathrm{~s} \\
1.2 \mathrm{MW} / 10 \mathrm{~s} \\
0.3 \mathrm{MW} / \mathrm{CW}\end{array}$ & $\begin{array}{l}1.55 \mathrm{MW} / 34.0 \%(27.6 \%) / 4 \mathrm{~ms} \\
0.64 \mathrm{MW} / 49.4 \%(28.4 \%) / 2 \mathrm{~ms}\end{array}$ & $\begin{array}{c}1.60 \mathrm{MW} / 36.6 \%(29.7 \%) / 0.5 \mathrm{~s} \\
1.53 \mathrm{MW} / 36.5 \%(29.7 \%) / 1.6 \mathrm{~s} \\
0.91 \mathrm{MW} / 40.7 \%(33.1 \%) / 1.8 \mathrm{~s} \\
0.22 \mathrm{MW} / 32.4 \%(24.3 \%) / 4500 \mathrm{~s}\end{array}$ \\
\hline
\end{tabular}

\section{Discussion}

In this section, we discuss the comparison between the experimental results and the calculation results. The comparison of beam current dependences of the experimental output power and the calculated output power of $28 \mathrm{GHz} 1 \mathrm{MW}$ gyrotron are shown in Fig.15. A set of the experimental output power was measured at output window with $\mathrm{V}_{\mathrm{k}}=80 \mathrm{kV}$. The calculations are adjusted to be the calculated window output power by the multiplied of the calculated cavity oscillation power with each pitch factor $\alpha$ of $1.0 \sim 1.5$ and the calculated transmission efficiency of $94.7 \%$ from the mode converter to the window. The calculated power increases with the $\alpha$ increase and is saturated in $\alpha=1.4$. The experimental output powers shown by closed circles increase with the increase in beam current $\mathrm{I}_{\mathrm{k}}$, but have tendency to saturate at higher $\mathrm{I}_{\mathrm{k}}$. In the comparison between the experimental and the calculation results, the electron beam may have $\alpha=1.4$ in $\mathrm{I}_{\mathrm{k}}<15 \mathrm{~A}$. The value of $\alpha$ decreases with increase in $\mathrm{I}_{\mathrm{k}}$, and may become 1.0 around $\mathrm{I}_{\mathrm{k}}=40 \mathrm{~A}$. In Fig. 15, the values in round brackets are the pitch factor $\alpha$ calculated by MIG simulation code with the experimental operation parameters. The $\mathrm{I}_{\mathrm{k}}$ dependences of $\alpha$ decrease and $\alpha$ spread increase are seen by MIG calculation results. This tendency of the $\alpha$ decrease and the $\alpha$ spread increase, which causes deterioration of efficiency or the out of optimization of operation parameter due to the $I_{a}$ increase, is one of the issues for the higher power and the higher efficiency gyrotron development.

The comparison of beam current dependences of the experimental output power and the calculated output power of $77 \mathrm{GHz} 1.5 \mathrm{MW}$ gyrotron are shown in Fig.16. The experimental output powers were measured at MOU output with $\mathrm{V}_{\mathrm{k}}=80 \mathrm{kV}$. The calculations are adjusted by the calculated cavity oscillation power with each pitch factor $\alpha$ of $0.9 \sim 1.3$, the calculated transmission efficiency of $97.5 \%$ from the mode converter to the window and the experimentally obtained transmission efficiency of MOU. The calculated power increases with the $\alpha$ increase and does not saturate up to $\alpha=1.3$. Like the $28 \mathrm{GHz} 1 \mathrm{MW}$ gyrotron, the experimental output powers shown by closed circles increase with the increase of beam current $I_{k}$ but have tendency to be saturated at the higher $I_{k}$. The electron beam may have $\alpha=1.3$ around $\mathrm{I}_{\mathrm{k}} \sim 20$ A. The pitch factor $\alpha$ decreases with the increase of $\mathrm{I}_{\mathrm{k}}$, and may become less than $\alpha=1.0$ at $\mathrm{I}_{\mathrm{k}}>60$ A. In Fig.16, the values in round brackets are the pitch factor $\alpha$ calculated by MIG simulation code with the experimental operation parameters of both the PRC and the NIFS test. The similar dependence of $\alpha$ decrease with increase of $I_{k}$ is seen by MIG calculation results, too. To avoid the $\alpha$ 
decrease and $\alpha$ spread increase in high beam current is one of the keys for the high power and the high efficiency gyrotron development.

Another issue is the stray RF caused by transmission loss in the gyrotron for high power and long pulse gyrotron operation. The stray RF in the gyrotron causes the heating of non-cooling or unexpected part. As a result, it causes outgassing and limitation of the pulse duration. In relatively higher frequency, the main factor of the stray RF is minute surface shaping and setting errors of the mode converter and/or transmission mirrors. At the relatively low frequency, the main factor of the stray RF is the diffraction due to the large RF beam divergence under the limits of mirror size and position. The issue of the stray RF is more difficult in the lower frequency region than in the higher frequency. On the $28 \mathrm{GHz} 1 \mathrm{MW}$ gyrotron, the influence of the stray RF is little within the design pulse duration of $1 \mathrm{sec}$. However, if the longer pulse operation is performed for $\mathrm{CW}$ operation, the influence of stray RF becomes important. As mentioned above, $77 \mathrm{GHz} 1.5 \mathrm{MW} \# 3$ gyrotron which has the improved designs of mode converter and mirrors has not faced sudden outgassing up to $0.2 \mathrm{MW}$ for $1200 \mathrm{sec}$., which is not the limit of the tube but limited by MOU outgassing, whereas the pulse duration of $77 \mathrm{GHz} \# 2$ gyrotron is limited at $0.2 \mathrm{MW} 300 \mathrm{sec}$. because of sudden outgassing in the tube. In the operation of the higher output power and longer pulse duration, it is quite important to watch carefully the influence of heating by the stray RF.
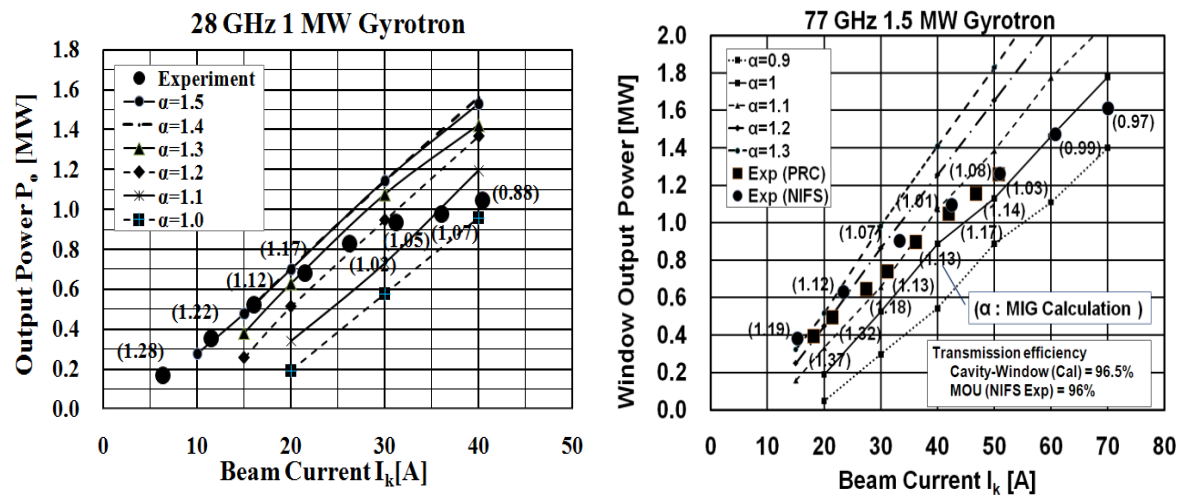

Fig.15 Comparison of beam current dependences of the experimental output power and the calculated output power of $28 \mathrm{GHz} 1 \mathrm{MW}$ gyrotron.

Fig.16 Comparison of beam current dependences of the experimental output power and the calculated output power of $77 \mathrm{GHz} 1.5 \mathrm{MW}$ gyrotron.

\section{Summary}

A new $28 \mathrm{GHz} 1 \mathrm{MW}$ gyrotron and three $77 \mathrm{GHz}$ over $1 \mathrm{MW}$ gyrotrons have been developed for ECRH systems of GAMMA10 and LHD NIFS under the joint program between NIFS and University of Tsukuba. In the initial test of the $28 \mathrm{GHz}$ tube with short pulse duration, the design target of $1 \mathrm{MW}$ output power was achieved and high oscillation efficiency of $40 \%$ was obtained. The third $77 \mathrm{GHz}$ tube has the improved design for the power up to $1.5 \mathrm{MW}$. The maximum output power of $1.6 \mathrm{MW}$ and the maximum total efficiency of $49.4 \%$ were obtained with short pulse duration at the MOU output. The long pulse test of two $77 \mathrm{GHz} 1 \mathrm{MW}$ and one $77 \mathrm{GHz} 1.5 \mathrm{MW}$ gyrotrons have been performed. 
The pulse width extended to $5 \mathrm{sec}$. with $1 \mathrm{MW}, 1800 \mathrm{sec}$. with $0.1 \mathrm{MW}, 1.6 \mathrm{sec}$. with 1.5 MW and so on. The limitation of the pulse duration which was caused by the stray RF heating has been improved by the design change of the mode converter and the mirrors of the 1.5MW \#3 gyrotron. Total injection power to LHD plasma has been achieved up to more than 3.1 MW. Comparing with the experimental results and the calculation results, it is found that the electron beam pitch factor $\alpha$ decrease with increase in beam current.

In conclusion, we have achieved more than $1 \mathrm{MW}$ operations of $28 \mathrm{GHz}$ and $77 \mathrm{GHz}$ gyrotrons and found some important issues for the further development of the high power and $\mathrm{CW}$ gyroton; The decrease in beam current in $\mathrm{CW}$ operation which causes the decrease of averaged power or the stop of oscillation. The decrease in pitch factor $\alpha$ with beam current causes the decrease in oscillation efficiency at higher beam current. The increase in $\alpha$ spread with beam current causes the decrease in CPD voltage and, as a result, the total efficiency limit, and brings the increase in the anode current that limits the operation at the higher efficiency region. The stray RF caused by transmission loss power heats up the non-cooling or unexpected part of the gyrotron inner surface and, as a result, gyrotron faces the outgassing or the limit of pulse duration. These issues could be overcome with appropriate design considerations fed back from the careful experimental studies

Acknowledgements The author thanks the members of the GAMMA10 group of the University of Tsukuba for their collaboration and valuable discussion during this study. This work is partially supported by NIFS Collaborative program (NIFS04KGM009, NIFS05KOAR006 and NIFS07KUGM009).

\section{References}

1. T. Saito et al., "High Power Electron Heating Experiments at the Plug Region of GAMMA 10”, J. Plasma Fusion Res. 81 (2005) 288-295 .

2. A. Mase et al., " Control of the radial electric field and of turbulent fluctuations in a tandem mirror plasma", Nucl. Fusion 31, (1991) 1725.

3. S. Moriyama et al., "Development and achievements on the high power ECRF system in JT-60U”, Nucl. Fusion 49 (2009) 085001.

4. M. Thumm, "MW gyrotron development for fusion plasma", Plasma Phys. Control. Fusion 45 (2003) A143-A161

5. T. Kariya et al., "Optimization of $28 \mathrm{GHz}$ Gyrotron Output Performance for ECRH Experiment of the GAMMA 10", Trans. of Fusion Science and Tech., 51, 2T (2006) 397-399.

6. T. Imai et al., "Upgrade Program of ECRH System for GAMMA 10", Trans. of Fusion Science and Tech., 51, $2 \mathrm{~T}$ (2006) 208-212.

7. T. Kariya et al., "Development of $28 \mathrm{GHz}$ and $77 \mathrm{GHz} 1 \mathrm{MW}$ Gyrotron for ECRH of magnetically confined plasma", Trans. of Fusion Science and Tech., 55, 2 T (2009) 91-94.

8. A. Kasugai, et al., "Long pulse operation of $170 \mathrm{GHz}$ ITER gyrotron by beam current control”, Fusion Engineering and Design, Vol.81 (2006) 2791-2796

9. K. Sakamoto et. al., "Achievement of robust high-efficiency 1MW oscillation in the hard-self-excitation region by a $170 \mathrm{GHz}$ continuous-wave gyrotron", Nat. Phys. 3 (2007) 411.

10. H. Takahashi et al., "The Development of a 77-GHz, 1-MW ECRH System for the Large Helical Device”, Fusion Science and Technology, Vol.57, No.1 (2010) 19-26 